\title{
BER Performance Analysis of Multistage PIC Scheme in Asynchronous DS-CDMA System over Unbalanced Multipath Fading Channels
}

\author{
Pei Xiao ${ }^{1}$, Erik Ström ${ }^{2}$, Rolando Carrasco ${ }^{1}$ \\ ${ }^{1}$ School of Electrical, Electronic and Computer Engineering \\ Univ. of Newcastle, NE1 7RU, United Kingdom \\ E-mail: pei.xiao, r.carrasco@ncl.ac.uk \\ ${ }^{2}$ Communication Systems Group Dept. of Signals and Systems \\ Chalmers Univ. of Tech., SE-412 96, Göteborg, Sweden \\ E-mail: erik.strom@chalmers.se
}

\begin{abstract}
In this paper, we provide a theoretical evaluation for the multistage parallel interference cancellation (PIC) scheme in a DS-CDMA system with orthogonal modulation and long scrambling codes. The studied system operates on the reverse link in a time-varying multipath Rayleigh fading channel. Unequal powers are assumed among different paths, which is usually the case in practical situations. The proposed analysis gives insight into the performance and capacity one can expect from the PIC based receivers under different situations.
\end{abstract}

\section{INTRODUCTION}

The system under study is an uncoded asynchronous DSCDMA system with orthogonal signalling formats. The transmitted chip sequence from a particular user is the concatenation of one of $M$ possible Walsh sequences (representing the transmitted symbol) and a long scrambling code. The Walsh (Hadamard) code is employed for combining the advantages of spreading and coding to achieve improved performance for spread spectrum (CDMA) systems.

The performance of interference canceler for short-code CDMA systems with BPSK signalling was investigated, e.g., in $[1,2]$. An adaptive multistage PIC scheme was analyzed in [1], and a closed form expression for BER performance is presented for the system operating over AWGN channels. In [2], an analytical BER expression for an adaptive multistage interference canceler was presented using an improved Gaussian approximation. The performance of orthogonal modulated DS-CDMA system with non-coherent and coherent combining was evaluated analytically in [3] and in [4] respectively.

BER performance of the multistage PIC scheme for the long-code CDMA system with orthogonal modulation was theoretically analyzed in [5]. To simplify computation, the multipath channels are assumed to have equal average power. Comparison with the simulated results shows that the analysis is fairly accurate. The presented analytical method provides an effective measure to predict BER performance and system capacity for the PIC scheme under investigation. In this paper, we extend the performance analysis to unbalanced multipath channels and derive BER expressions when different paths have unequal powers.

\section{System Model And Receiver AlgORITHMS}

System model is only briefly described here. Refer to $[5,6]$ for a more detailed description. The $k^{\text {th }}$ user $j^{\text {th }}$ transmitted symbol is denoted by $i_{k}(j) \in\{1,2, \cdots, M\}$, and is mapped into $\mathbf{w}_{k}(j) \in\left\{\mathbf{w}_{1}, \mathbf{w}_{2}, \cdots, \mathbf{w}_{m}, \cdots, \mathbf{w}_{M}\right\}$, which is one of the $M$ orthogonal signal alternatives. The Walsh codeword $\mathbf{w}_{k}(j) \in\{+1,-1\}^{M}$, is then repetition encoded into $\mathbf{s}_{k}(j) \in\{+1,-1\}^{N}$ so that each bit of the Walsh codeword is spread (repetition coded) into $N_{c}=N / M$ chips, and each Walsh symbol is represented by $N$ chips. The Walsh sequence $\mathbf{s}_{k}(j)$ is then scrambled (randomized) by a scrambling code unique to each user to form the transmitted chip sequence $\mathbf{a}_{k}(j)=\mathbf{C}_{k}(j) \mathbf{s}_{k}(j) \in\{+1,-1\}^{N}$ where $\mathbf{C}_{k}(j) \in\{-1,0,+1\}^{N \times N}$ is a diagonal matrix whose diagonal elements correspond to the scrambling code for the $k^{t h}$ user's $j^{\text {th }}$ symbol.

The baseband signal is formed by pulse amplitude modulating $\mathbf{a}_{k}(j)$ with a unit-energy rectangular chip waveform $\psi(t)$. The baseband signal is then multiplied with a carrier and transmitted over a Rayleigh fading channel with $L_{k}$ resolvable paths, having time-varying channel gains $h_{k, 1}(t), h_{k, 2}(t), \ldots, h_{k, L_{k}}(t)$ and delays $\tau_{k, 1}, \tau_{k, 2}, \ldots, \tau_{k, L_{k}}$. The received signal is the sum of $K$ users' signals plus additive white complex Gaussian noise $n(t)$. The received vector corresponding to the $k^{t h}$ user's $j^{\text {th }}$ symbol after frequency down-conversion and chip matched filtering is denoted as $\mathbf{r}(k, j)$ and can be written in vector form as

$$
\begin{aligned}
& \mathbf{r}(k, j)=\mathbf{A}(k, j) \mathbf{h}(j)+\mathbf{n}(k, j) \\
& =\mathbf{X}_{k}(j) \mathbf{h}_{k}(j)+\operatorname{ISI}(\mathrm{k}, \mathrm{j})+\operatorname{MAI}(\mathrm{k}, \mathrm{j})+\mathbf{n}(\mathrm{k}, \mathrm{j}) \in \mathbb{C}^{\mathrm{N}_{\mathrm{k}}}
\end{aligned}
$$

where the columns of the matrix $\mathbf{A}(k, j)$ are delayed versions of the transmitted chip sequences $\mathbf{a}_{k}(j)$ for $k=$ $1,2, \cdots, K$, one column per path. The length of the processing window $N_{k}$, is larger than the symbol interval $N$ to account for the asynchronous and multipath nature of the channel. The columns are weighted together by $\mathbf{h}(j)$, whose elements are the path gains of all users' paths. The received vector $\mathbf{r}(k, j)$ can be written as the sum of four terms: the signal of interest $\mathbf{X}_{k}(j) \mathbf{h}_{k}(j)$, the intersymbol interference (ISI), the multiple access interference (MAI), and the noise represented by $\mathbf{n}(k, j)$, which is a vector of complex Gaussian noise 1 samples with zero mean and variance $N_{0}$. The vector 


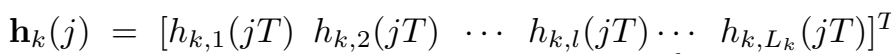
corresponds to the channel gains of the $k^{t h}$ user's paths, it is a part of $\mathbf{h}(j)$.

The task of the receiver is to detect the information bits from all users, i.e., detect $i_{k}(j)$ for $k=1,2, \ldots, K$, $j=1,2, \ldots, L_{b}\left(L_{b}\right.$ is the block length) given the observation $\mathbf{r}(k, j)$. The decision on the $k^{t h}$ user's $j^{\text {th }}$ symbol, is found as $\hat{i}_{k}(j)=\arg \max _{m \in\{1,2, \cdots, M\}} z_{k}(m)$ where $z_{k}(m)$ is the decision statistic from symbol matched filter (MF) or multiuser detector (interference canceler in our case), based on the condition that the $m^{t h}$ Walsh symbol is transmitted from user $k$. With conventional MF, the soft decision is formed by correlating the received signal with the $M$ possible transmitted waveforms

$$
z_{k}^{\mathrm{MF}}(m)=\sum_{l=1}^{L_{k}}\left|\mathbf{x}_{k, l, m}^{*}(j) \mathbf{r}(k, j)\right|^{2}
$$

where $\mathbf{x}_{k, l, m}$ denotes the transmitted chip sequence due to the $k^{t h}$ user's $j^{t h}$ symbol from the $l^{t h}$ path based on the hypothesis that the $m^{t h}$ Walsh symbol is transmitted.

This simple scheme is particularly useful in the beginning of the detection process when the estimates of the fading channel are lacking, we must therefore carry out the detection in a non-coherent manner. However, it has poor performance in multiuser environment since it considers multiple access interference (MAI) as additive noise and the knowledge about MAI is not exploited in any way. An effective tool to increase the capacity of interference-limited CDMA systems is multiuser detection (MUD), a method of jointly detecting all the users in the system. Among different MUD techniques, the multistage interference cancellation schemes are known to be simple and effective for mitigation of MAI in long-code DS-CDMA systems. For the purpose of this study, we consider the PIC scheme introduced in [6].

The basic principle is that once the transmitted signals are estimated for all the users at the previous iteration, interference can be removed by subtracting the estimated signals of the interfering users from the received signal $\mathbf{r}(k, j)$ to form a new signal vector $\mathbf{r}^{\prime}(k, j)$ for demodulating the signal transmitted from user $k$, i.e.,

$$
\mathbf{r}^{\prime}(k, j)=\mathbf{r}(k, j)-\hat{\mathbf{A}}(k, j) \hat{\mathbf{h}}(j)+\hat{\mathbf{X}}_{k}(j) \hat{\mathbf{h}}_{k}(j)
$$

where $\mathbf{r}^{\prime}(k, j) \in \mathbb{C}^{N_{k}}$ denotes the interference canceled version of $\mathbf{r}(k, j)$ after subtracting the contributions from all the other users using decision feedback at the previous stage. The vector $\hat{\mathbf{A}}(k, j) \hat{\mathbf{h}}(j)$ represents the estimated contribution from all the users. The vector $\hat{\mathbf{X}}_{k}(j) \hat{\mathbf{h}}_{k}(j)$ is the estimated contribution from all paths of user $k$. The soft decision with PIC is formed as

$$
z_{k}^{\mathrm{PIC}}(m)=\operatorname{Re}\left\{\hat{\mathbf{h}}_{k}^{*}(j) \mathbf{X}_{k, m}^{*} \mathbf{r}^{\prime}(k, j)\right\}
$$

where $\mathbf{X}_{k, m}=\left[\begin{array}{llll}\mathbf{x}_{k, 1, m} & \mathbf{x}_{k, 2, m} & \cdots & \mathbf{x}_{k, L_{k}, m}\end{array}\right]$.

\section{Theoretical Analysis}

\section{A. Performance analysis for non-coherent first stage}

To evaluate the probability of error, without loss of generality, let us assume that the $j^{t h}$ symbol transmitted from the $k^{\text {th }}$ user is the first Walsh symbol and the channel gain remains constant during one symbol interval. The decision statistic expressed in (2) can be reformed as

$$
z_{k}^{\mathrm{MF}}(m)= \begin{cases}\sum_{l=1}^{L_{k}}\left|\mathcal{D}_{k, l}+\mathcal{M}_{k, l}+\mathcal{I}_{k, l}+\mathcal{N}_{k, l}\right|^{2}, & \text { if } m=1 \\ \sum_{l=1}^{L_{k}}\left|\mathcal{M}_{k, l}+\mathcal{I}_{k, l}+\mathcal{N}_{k, l}\right|^{2}, & \text { if } m \neq 1\end{cases}
$$

where $\mathcal{D}_{k, l}=N h_{k, l}$, and $\mathcal{M}_{k, l}, \mathcal{I}_{k, l}, \mathcal{N}_{k, l}$ stand for the desired signal, contribution from MAI, ISI, and noise, respectively. It was justified in [5] that Gaussian approximation is accurate to model MAI and ISI sequences as well as the elements of each interference sequence in long-code systems. For the $k^{t h}$ user's $l^{t h}$ receiver branch, the interference plus noise variance is $\sigma^{2}=\sigma_{\mathcal{M}}^{2}+\sigma_{\mathcal{I}}^{2}+\sigma_{\mathcal{N}}^{2}$, where $\sigma_{\mathcal{M}}^{2}=$ $\operatorname{var}\left[\mathcal{M}_{k, l}\right]=\mathrm{E}\left[\left|\mathcal{M}_{k, l}\right|^{2}\right], \quad \sigma_{\mathcal{I}}^{2}=\operatorname{var}\left[\mathcal{I}_{k, l}\right]=\mathrm{E}\left[\left|\mathcal{I}_{k, l}\right|^{2}\right], \quad \sigma_{\mathcal{N}}^{2}=$ $\operatorname{var}\left[\mathcal{N}_{k, l}\right]=\mathrm{E}\left[\left|\mathcal{N}_{k, l}\right|^{2}\right]$. Note that $\sigma^{2}, \sigma_{\mathcal{M}}^{2}, \sigma_{\mathcal{I}}^{2}$ depend on $k$ and $l$, which is not explicitly indicated in order to simplify notations. The noise variance can be easily computed as $\sigma_{\mathcal{N}}^{2}=\sum_{n=1}^{n=N} N_{0}=N N_{0}$. According to [5], the variance of the MAI and ISI for chip synchronous systems can be expressed as

$$
\begin{aligned}
\sigma_{\mathcal{M}}^{2} & =\sum_{\substack{s=1 \\
s \neq k}}^{K} \sum_{i=1}^{L_{s}} \sum_{n=1}^{N} \mathrm{E}\left[\left\|h_{s, i}\right\|^{2}\right]=N \sum_{\substack{s=1 \\
s \neq k}}^{K} \sum_{i=1}^{L_{s}} P_{s, i} \\
\sigma_{\mathcal{I}}^{2} & =\sum_{\substack{i=1 \\
i \neq l}}^{L_{k}} \sum_{n=1}^{N} \mathrm{E}\left[\left\|h_{k, i}\right\|^{2}\right]=N \sum_{\substack{i=1 \\
i \neq l}}^{L_{k}} P_{k, i}
\end{aligned}
$$

The expressions for chip asynchronous systems can be analyzed similarly. When each path has unequal power, i.e., $P_{k, 1} \neq P_{k, 2} \neq \cdots \neq P_{k, L_{k}}$, the decision statistic expressed in (4) can be formed as

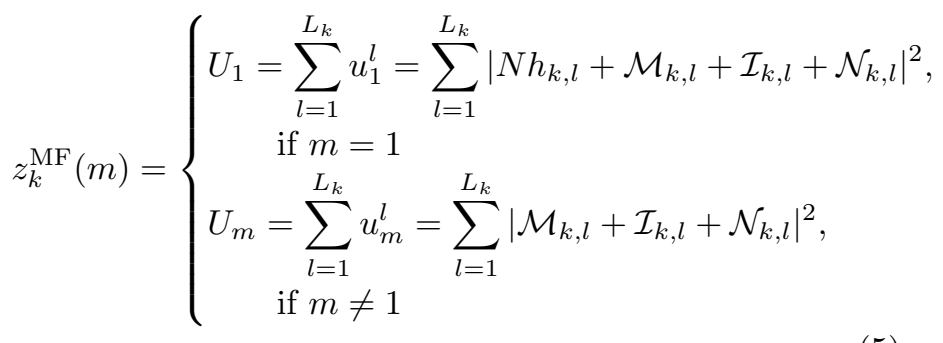

In case $m=1$, each term $u_{1}^{l}=\left|N h_{k, l}+\mathcal{M}_{k, l}+\mathcal{I}_{k, l}+\mathcal{N}_{k, l}\right|^{2}$ is an independent central chi-square distributed random variable with 2 degrees of freedom and characteristic function $\psi_{u_{1}^{l}}(j v)=\left(1-j v \gamma_{l}\right)^{-1}$, where $\gamma_{l}=\mathrm{E}\left[\mid N h_{k, l}+\mathcal{M}_{k, l}+\right.$ $\left.\mathcal{I}_{k, l}+\left.\mathcal{N}_{k, l}\right|^{2}\right]=N^{2} P_{k, l}+\sigma_{\mathcal{M}}^{2}+\sigma_{\mathcal{I}}^{2}+\sigma_{\mathcal{N}}^{2}$. As a consequence of the statistical independence of $u_{1}^{l}, l=1,2, \ldots, L_{k}$, the characteristic function of $U_{1}$ is

$$
\psi_{U_{1}}(j v)=\prod_{l=1}^{L_{k}}\left(1-j v \gamma_{l}\right)^{-1}=\sum_{l=1}^{L_{k}} A_{l}\left(1-j v \gamma_{l}\right)^{-1}
$$


Taking the Fourier transform of (6), we obtain the pdf of $U_{1}$ as

$$
p\left(U_{1}\right)=\sum_{l=1}^{L_{k}} \frac{A_{l}}{\gamma_{l}} \exp \left(-\frac{U_{1}}{\gamma_{l}}\right) ; \quad 0 \leq U_{1} \leq \infty
$$

Similarly,

$$
p\left(U_{m}\right)=\sum_{l=1}^{L_{k}} \frac{B_{l}}{\beta_{l}} \exp \left(-\frac{U_{m}}{\beta_{l}}\right) ; \quad 0 \leq U_{m} \leq \infty, \quad m \neq 1
$$

where $\beta_{l}=\mathrm{E}\left[\left|\mathcal{M}_{k, l}+\mathcal{I}_{k, l}+\mathcal{N}_{k, l}\right|^{2}\right]=\sigma_{\mathcal{M}}^{2}+\sigma_{\mathcal{I}}^{2}+\sigma_{\mathcal{N}}^{2}$, and $B_{l}=\prod_{i=1, i \neq l}^{i=L_{k}}\left(1-\frac{\beta_{i}}{\beta_{l}}\right)^{-1}$.

The probability of making correct symbol decision can be computed as

$$
\begin{aligned}
\mathcal{P}_{\mathrm{c}, k} & =P_{r}\left(U_{2}<U_{1}, U_{3}<U_{1}, \cdots, U_{M}<U_{1}\right) \\
& =\int_{0}^{\infty}\left[P_{r}\left(U_{2}<U_{1}\right)\right]^{M-1} p\left(U_{1}\right) d U_{1} \\
P_{r}\left(U_{2}<U_{1}\right) & =\int_{0}^{U 1} p\left(U_{2}\right) d U_{2}=\sum_{l=1}^{L_{k}} B_{l}\left[1-\exp \left(-\frac{U_{1}}{\beta_{l}}\right)\right]
\end{aligned}
$$

Therefore, the BER for non-coherent first stage in unequal power multipath system is derived as

$$
\begin{aligned}
\mathcal{P}_{\mathrm{c}, k}= & \int_{0}^{\infty}\left(\sum_{l=1}^{L_{k}} B_{l}\left[1-\exp \left(-\frac{U_{1}}{\beta_{l}}\right)\right]\right)^{M-1} \\
& \cdot \sum_{l=1}^{L_{k}} \frac{A_{l}}{\gamma_{l}} \exp \left(-\frac{U_{1}}{\gamma_{l}}\right) d U_{1} \\
\mathcal{P}_{\mathrm{b}, k}= & \frac{M}{2(M-1)} \mathcal{P}_{\mathrm{e}, k}=\frac{M}{2(M-1)}\left(1-\mathcal{P}_{\mathrm{c}, k}\right)
\end{aligned}
$$

\section{B. Performance analysis for multistage PIC}

Let us assume that the first Walsh symbol was transmitted from the $k^{t h}$ user. The decision statistic expressed in (3) can be reformed after $p$ stages of cancellations

$$
\begin{aligned}
& z_{k}^{(p)}(m)=\sum_{l=1}^{L_{k}} \operatorname{Re}\left\{\hat{h}_{k, l}^{*} \mathbf{x}_{k, l, m} \mathbf{r}^{\prime}\right\} \\
& = \begin{cases}\sum_{l=1}^{L_{k}} d_{k, l}+\mathcal{M}_{k, l}^{(p)}+\mathcal{I}_{k, l}^{(p)}+n_{k, l}=d+n_{1}^{(p)}, & \text { if } m=1 \\
\sum_{l=1}^{L_{k}} \mathcal{M}_{k, l}^{(p)}+\mathcal{I}_{k, l}^{(p)}+n_{k, l}=n_{m}^{(p)}, & \text { if } m \neq 1\end{cases}
\end{aligned}
$$

where $d=\sum_{l=1}^{L_{k}} d_{k, l}=N \sum_{l=1}^{L_{k}} h_{k, l} \hat{h}_{k, l}^{*}$ is the desired signal. Assume accurate channel estimation, i.e., $\hat{h}_{k, l} \approx h_{k, l}$, then $d=\sum_{l=1}^{l=L_{k}} d_{k, l} \approx N \sum_{l=1}^{l=L_{k}}\left|h_{k, l}\right|^{2}$. The noise component for the $l^{t h}$ diversity branch is denoted by $n_{k, l}$. The contributions of MAI and ISI to the $l^{\text {th }}$ diversity branch at the $p^{t h}$ stage are denoted by $\mathcal{M}_{k, l}^{(p)}$ and $\mathcal{I}_{k, l}^{(p)}$ respectively. Using the Gaussian approximation, $n_{1}^{(p)}, n_{2}^{(p)}, \ldots, n_{M}^{(p)}$ are zero-mean statistically independent Gaussian random variables with equal variance $\left(\sigma^{2}\right)^{(p)} / 2$, where $\left(\sigma^{2}\right)^{(p)}$ denotes the interference plus noise variance at the $p^{\text {th }}$ stage, and it is defined as $\left(\sigma^{2}\right)^{(p)}=\left(\sigma_{\mathcal{M}}^{2}\right)^{(p)}+\left(\sigma_{\mathcal{I}}^{2}\right)^{(p)}+\sigma_{n}^{2}$.

Let us denote $\mathcal{P}_{\mathrm{c}, k}^{(p)}(x)$ as the probability that the receiver makes correct symbol decision for user $k$ at the $p^{\text {th }}$ stage conditioned on $x$, which is defined as $x=\frac{d}{\sigma^{(p)}}$. It is the probability that $z_{k}^{(p)}(1)=d+n_{1}^{(p)}$ is larger than each of the other $M-1$ outputs $z_{k}^{(p)}(2)=n_{2}^{(p)}, z_{k}^{(p)}(3)=$ $n_{3}^{(p)}, \ldots, z_{k}^{(p)}(M)=n_{M}^{(p)}[7]$ :

$\mathcal{P}_{\mathrm{c}, k}^{(p)}(x)=\frac{1}{\sqrt{2 \pi}} \int_{-\infty}^{\infty}[1-Q(y)]^{M-1} \exp \left[-\frac{1}{2}(y-\sqrt{2} x)^{2}\right] d y$

where the function $Q(x)$ is defined as $Q(x)=\frac{1}{2} \operatorname{erfc}\left(\frac{x}{\sqrt{2}}\right)$.

The noise term does not change between iterations and can be computed as $\sigma_{n}^{2}=N N_{0} \sum_{l=1}^{L_{k}}\left|h_{k, l}\right|^{2}$. However, the variance of MAI and ISI change at each iteration due to interference cancellation. At the $p^{t h}$ stage, the probability of correct cancellation is $\mathcal{P}_{\mathrm{c}, s}^{(p-1)}=1-\mathcal{P}_{\mathrm{e}, s}^{(p-1)}$, where the interfering user $s=1, \ldots, K$, and $s \neq k$. For chip synchronous systems, the variance of MAI from all the diversity branches of user $k$ is [5]

$$
\left(\sigma_{\mathcal{M}}^{2}\right)^{(p)}=\sum_{l=1}^{L_{k}}\left(\sigma_{\mathcal{M}}^{2}\right)_{l}^{(p)}=\sum_{l=1}^{L_{k}}\left|h_{k, l}\right|^{2} 2 N \sum_{\substack{s=1 \\ s \neq k}}^{K} \sum_{i=1}^{L_{s}} \mathcal{P}_{\mathrm{e}, s}^{(p-1)} P_{s, i}
$$

Denote $p_{k, i}$ and $p_{k, l}$ as integer part of the delays $\tau_{k, i}$ and $\tau_{k, l}$. The variance the total ISI term for user $k$ is computed as $[5]$

$\left(\sigma_{\mathcal{I}}^{2}\right)^{p}=\sum_{l=1}^{L_{k}}\left|h_{k, l}\right|^{2} \sum_{\substack{i=1 \\ i \neq l}}^{L_{k}}\left[N+\left(2 \mathcal{P}_{\mathrm{e}, k}^{(p-1)}-1\right)\left|p_{k, i}-p_{k, l}\right|\right] P_{k, i}$

Based on the above analysis, we derive the total noise plus interference variance as $\left(\sigma^{2}\right)^{(p)}=\sigma_{n}^{2}+\left(\sigma_{\mathcal{I}}^{2}\right)^{(p)}+\left(\sigma_{\mathcal{M}}^{2}\right)^{(p)}=$ $\sum_{l=1}^{L_{k}}\left|h_{k, l}\right|^{2}\left(\alpha_{l}^{2}\right)^{(p)}$, and $\left(\alpha_{l}^{2}\right)^{(p)}$ is defined as

$$
\begin{aligned}
\left(\alpha_{l}^{2}\right)^{(p)}=N & N_{0}+2 N \sum_{\substack{s=1 \\
s \neq k}}^{K} \sum_{i=1}^{L_{s}} \mathcal{P}_{\mathrm{e}, s}^{(p-1)} P_{s, i} \\
& +\sum_{\substack{i=1 \\
i \neq l}}^{L_{k}}\left[N+\left(2 \mathcal{P}_{\mathrm{e}, k}^{(p-1)}-1\right)\left|p_{k, i}-p_{k, l}\right|\right] P_{k, i}
\end{aligned}
$$

In case each path has unequal power, the variable $x=$ $\frac{d}{\sigma^{(p)}}$ is formed as

$$
x=\frac{d}{\sigma^{(p)}}=\frac{N \sum_{l=1}^{L_{k}}\left|h_{k, l}\right|^{2}}{\sqrt{\sum_{l=1}^{L_{k}}\left|h_{k, l}\right|^{2}\left(\alpha_{l}^{2}\right)^{(p)}}}
$$

The pdf of $x$ is difficult to derive under such a circumstance because the numerator and denominator are not independent. However, if the self interference is small compared to noise and MAI, e.g., when the number of users 
$K$ is much bigger than the number of paths $L_{k}$, which is usually the case, or when SNR is low, we can approximate $\left(\alpha_{1}^{2}\right)^{(p)} \approx\left(\alpha_{2}^{2}\right)^{(p)} \approx \cdots \approx\left(\alpha_{L_{k}}^{2}\right)^{(p)} \approx\left(\alpha^{2}\right)^{(p)}$, then we can denote $z=x^{2}=\sum_{l=1}^{l=L_{k}} z_{l} \approx \frac{N^{2}}{\left(\alpha^{2}\right)^{(p)}} \sum_{l=1}^{l=L_{k}}\left|h_{k, l}\right|^{2}$, and each term $z_{l} \approx \frac{N^{2}}{\left(\alpha^{2}\right)^{(p)}}\left|h_{k, l}\right|^{2}$ is an independent central chisquare distributed random variable with 2 degrees of freedom and characteristic function $\psi_{z_{l}}(j v)=\left(1-j v \gamma_{l}^{(p)}\right)^{-1}$, where $\gamma_{l}^{(p)}=\frac{N^{2}}{\left(\alpha^{2}\right)^{(p)}} \mathrm{E}\left[\left|h_{k, l}\right|^{2}\right]=\frac{N^{2} P_{k, l}}{\left(\alpha^{2}\right)^{(p)}}$. As a consequence of the statistical independence of $z_{l}, l=1,2, \ldots, L_{k}$, the characteristic function of $z$ is

$$
\begin{aligned}
\psi_{z}(j v) & =\prod_{l=1}^{L_{k}}\left(1-j v \gamma_{l}^{(p)}\right)^{-1} \\
& =\sum_{l=1}^{L_{k}}\left[\prod_{i=1, i \neq l}^{L_{k}}\left(1-\frac{\gamma_{i}^{(p)}}{\gamma_{l}^{(p)}}\right)^{-1}\right]\left(1-j v \gamma_{l}^{(p)}\right)^{-1} \\
& =\sum_{l=1}^{L_{k}} A_{l}^{(p)}\left(1-j v \gamma_{l}^{(p)}\right)^{-1}
\end{aligned}
$$

where $A_{l}^{(p)}=\prod_{i=1, i \neq l}^{l=L_{k}}\left(1-\gamma_{i}^{(p)} / \gamma_{l}^{(p)}\right)^{-1}$. Taking the Fourier transform of (10), we obtain the pdfs of $z$ and $x$ as

$$
\begin{aligned}
& p(z)=\sum_{l=1}^{L_{k}} \frac{A_{l}^{(p)}}{\gamma_{l}^{(p)}} \exp \left[-\frac{z}{\gamma_{l}^{(p)}}\right] ; 0 \leq z \leq \infty \\
& p(x)=2 x \sum_{l=1}^{L_{k}} \frac{A_{l}^{(p)}}{\gamma_{l}^{(p)}} \exp \left[-\frac{x^{2}}{\gamma_{l}^{(p)}}\right] ; \quad 0 \leq x \leq \infty
\end{aligned}
$$

To obtain the error probability when $x$ is random, we must average $\mathcal{P}_{\mathrm{c}, k}^{(p)}(x)$ given in (9) over the distribution of $x$, i.e.,

$$
\mathcal{P}_{\mathrm{c}, k}^{(p)}=\mathrm{E}\left[\mathcal{P}_{\mathrm{c}, k}^{(p)}(x)\right]=\int_{0}^{\infty} \mathcal{P}_{\mathrm{c}, k}^{(p)}(x) p(x) d x
$$

Without carrying out the integration, $\mathcal{P}_{\mathrm{c}, k}^{(p)}$ can be approximated according to [8] by

$\mathcal{P}_{\mathrm{c}, k}^{(p)} \approx \frac{2}{3} \mathcal{P}_{\mathrm{c}, k}^{(p)}\left(\mu_{x}\right)+\frac{1}{6} \mathcal{P}_{\mathrm{c}, k}^{(p)}\left(\mu_{x}+\sqrt{3} \sigma_{x}\right)+\frac{1}{6} \mathcal{P}_{\mathrm{c}, k}^{(p)}\left(\mu_{x}-\sqrt{3} \sigma_{x}\right)$

where $\mu_{x}$ and $\sigma_{x}$ are the mean and standard deviation of $x$

$$
\begin{aligned}
\mu_{x} & =\mathrm{E}[x]=\int_{0}^{\infty} x p(x) d x=\frac{\sqrt{\pi}}{4} \sum_{l=1}^{L_{k}} A_{l}^{(p)} \sqrt{\gamma_{l}^{(p)}} \\
\mathrm{E}\left[x^{2}\right] & \approx \mathrm{E}\left[\frac{N^{2}}{\left(\alpha^{2}\right)^{(p)}} \sum_{l=1}^{L_{k}}\left|h_{k, l}\right|^{2}\right]=\frac{N^{2}}{\left(\alpha^{2}\right)^{(p)}} \sum_{l=1}^{L_{k}} P_{k, l} \\
\sigma_{x} & =\sqrt{\mathrm{E}\left[x^{2}\right]-\mu_{x}^{2}}
\end{aligned}
$$

The average bit error rate at the $p^{\text {th }}$ cancellation stage is

$$
\mathcal{P}_{\mathrm{b}, k}^{(p)}=\frac{M}{2(M-1)} \mathcal{P}_{\mathrm{e}, k}^{(p)}=\frac{M}{2(M-1)}\left(1-\mathcal{P}_{\mathrm{c}, k}^{(p)}\right)
$$

It is initialized with $\mathcal{P}_{\mathrm{b}, k}^{(1)}$, the BER of the first noncoherent stage, which is computed according to (7).

\section{Performance Comparison And Analytical Results}

The results of theoretical analysis as well as its comparison with simulation results are presented in this section. It is assumed that each user transmits one of $M=8$ Walsh codes spread to a total length of $N=64$ chips. The effective spreading of the system is $N / \log _{2} M=64 / 3$ chips per bit. Different users are separated by different scrambling codes $\mathbf{C}_{k}(j)$ which are random, and differ from symbol to symbol. The noise variance $N_{0}$ and $\mathbf{C}_{k}$ as well as delays $\tau_{k, 1}, \tau_{k, 2}, \ldots, \tau_{k, L_{k}}$ are assumed to be known to the receiver. Channels are independent Rayleigh fading channels. The normalized Doppler frequency is assumed to be $f_{d} T=0.01$. The number of multipath channels $L_{k}$ is set to be the same for all $k$, i.e., $\left(L_{k}=L\right)$. Power control is assumed so that the average received power is equal for all users and each user has unity gain.

Figure 1 shows the comparison between analytical and simulated results for a 12-user system. The number of multipath channels $L_{k}$ is set to be 4 . To simplify the simulations, the system is assumed to be chip-synchronous, i.e., all path delays are assumed to be multiples of $T_{c}$. However, the system is asynchronous on the symbol level. The power difference between different paths is set to be $\Delta P_{k, l}=P_{k, 4}-P_{k, 3}=P_{k, 3}-P_{k, 2}=P_{k, 2}-P_{k, 1}=0.005 \mathrm{~dB}$. Channel estimation is conducted with the Maximum Likelihood algorithm introduced in [6]. The simulation results are averaged over random distributions of fading, noise, delay, and scrambling code through numerous Monte-Carlo runs. One can see from Figure 1 that the simulated curve precisely matches the theoretical one for the first noncoherent stage. The analysis starts to deviate slightly from simulations, but is still fairly accurate, after the first noncoherent stage. This validates the theoretical analysis and its accuracy. Both simulation and analysis indicate that it takes 4 or 5 stages (excluding the first non-coherent stage) for the PIC to converge.

To study the impact of the power difference between different paths on the performance of the PIC scheme, $\Delta P_{k, l}$ is set to $0,3,6 \mathrm{~dB}$, respectively, in Figure 2. The results show that the PIC works the best and obtains the most diversity gain when all the branches have equal power, i.e., when $\Delta P_{k, l}=0$. The bigger deviation in power, the worse performance it gets.

Fast and accurate power control is hard to achieve in practice. It is therefore important to study the near-far robustness of the PIC algorithm. Figure 3 shows its analytical BER performance as a function of near-far ratio, which refers to the difference between the power of each of interfering user (it is assumed that $P_{2}=P_{3}=\cdots=P_{m}=$ $\cdots=P_{K}$ ), and the power of the desired user $P_{1}$ (the first user is the user of interest). Figure 3 shows that the PIC scheme is not sensitive to the variations in the interfering signal strengths and is near-far resistant after the system reaches convergence. It also shows that the near-far robustness of the PIC scheme comes from interference cancella4 


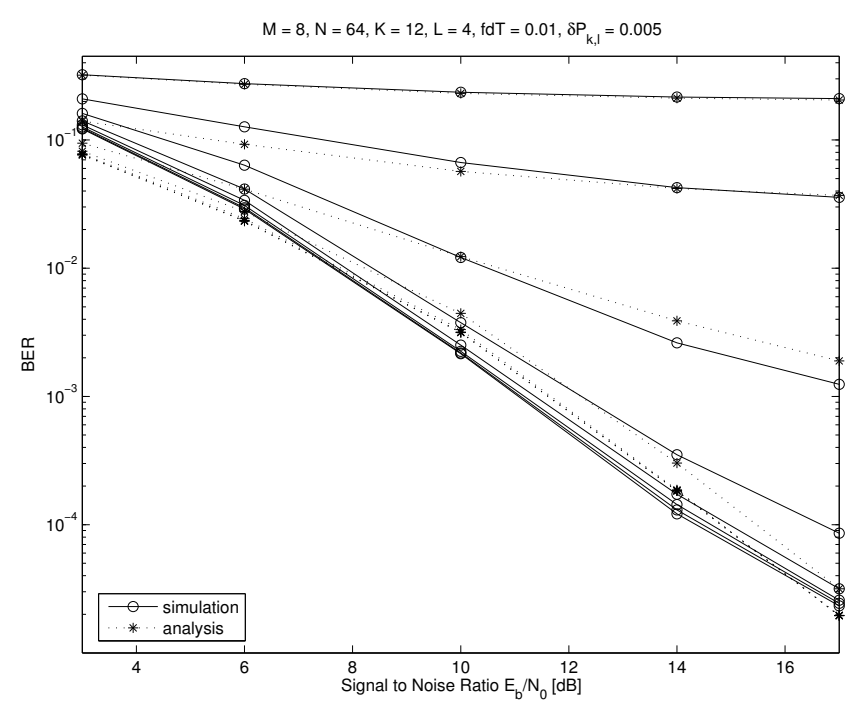

Fig. 1. Comparison between simulation and analysis. Topmost curve represents non-coherent first stage and the second curve from top represents the first stage PIC, the bottommost curve represents the $7^{\text {th }}$ stage PIC.

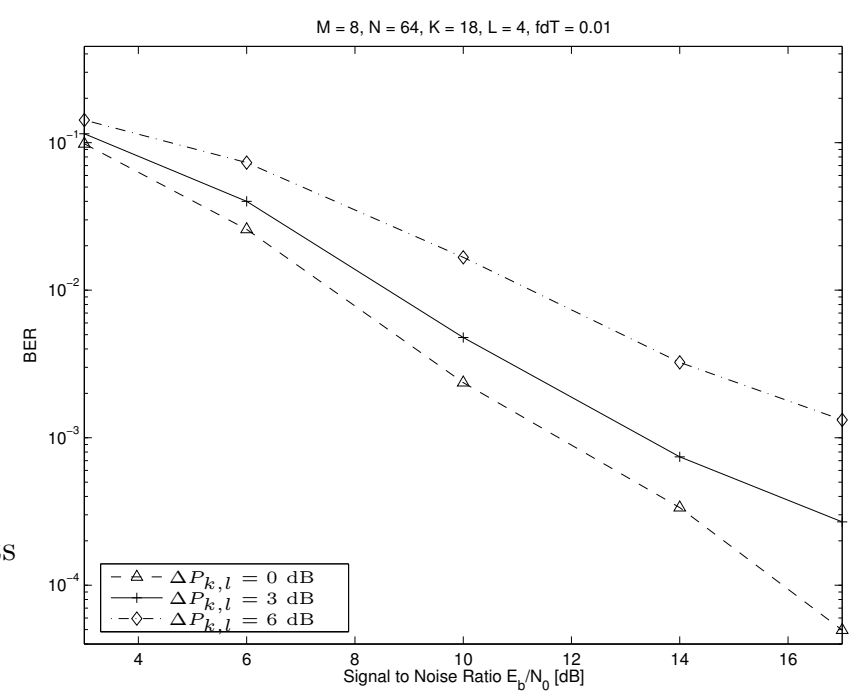

Fig. 2. PIC performance for unequal power diversity branches, the curves are plotted for the $7^{\text {th }}$ stage.

of near-far problem, which will gradually vanish as the iteration goes on and the system reaches convergence. The rationale is that the error probability for strong interfering users is very low due to their high signal power level, we therefore have better chance to make correct cancellation and cancel their contributions, which greatly alleviates the near-far effect. It is also shown that the PIC scheme converges after 4 or 5 stages (excluding the first non-coherent stage).

\section{Conclusions}

BER performance of the multistage PIC scheme for the asynchronous DS-CDMA system employing orthogonal modulation and long spreading codes is theoretically analyzed in this paper. In particular, the BER expressions are

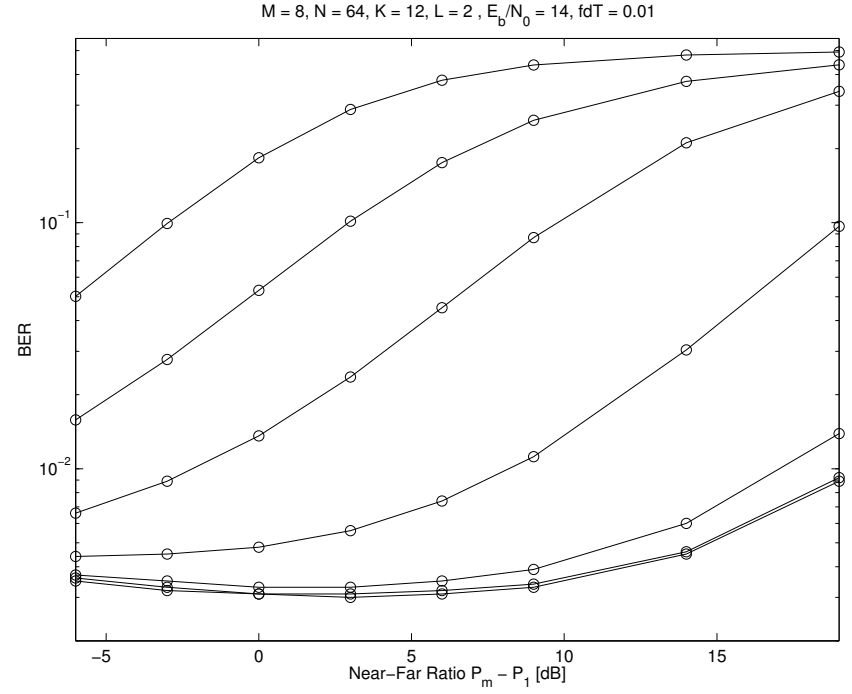

Fig. 3. Near-far effect for PIC: analytical BER vs. NFR at different stages of PIC.

derived for the system operating in unbalanced multipath Rayleigh fading channels where different paths have unequal power. Comparison with the simulated results shows that the analysis is fairly accurate. The multipath diversity gain and near-far effect of the PIC scheme are examined using this analytical approach. The study shows that the PIC is near-far resistant, and can be used in practical systems even when strict power control is hard to obtain. We also learned that the PIC scheme achieves the best performance in presence of equal power among different diversity branches. Unbalanced multipaths lead to less diversity gain.

\section{REFERENCES}

[1] A. Kaul, B. Woerner. "Analytic limits on performance of adaptive multistage interference cancellation for CDMA". Electronics Letters, vol. 30, no. 25, pp. 2093-2095, Dec. 1994.

[2] R. Buehrer, B. Woerner. "Analysis of adaptive multistage interference cancellation for CDMA using an improved Gaussian approximation". IEEE Transactions on Communications, vol. 44, no. 10, pp. 1308-1321, Oct. 1996.

[3] A. Naguib, A. Paulraj. "Performance of Wireless CDMA with Mary orthogonal modulation and cell site antenna arrays". IEEE Journal on Selected Areas Communications, vol. 14, no. 9, pp. 1770-1783, Dec. 1996.

[4] J. Thompson, P. Grant, B. Mulgrew. "Algorithms for coherent diversity combining of M-ary orthogonal signals". IEEE Journal on Selected Areas in Communications, vol. 17, no. 11, pp. 18861899, Nov. 1999.

[5] P. Xiao, E. Ström. "BER performance analysis of multistage PIC scheme in an asynchronous DS-CDMA system with long scrambling codes". In Proc. IEEE Vehicular Technology Conference, vol. 2, pp. 952-957, May 2004.

[6] E. Ström, S. Miller. "Iterative demodulation and channel estimation of orthogonal signalling formats in asynchronous DS-CDMA systems". IEICE Transactions on Electronics, vol. E85-C, no. 3, pp. 442-451, March 2002

[7] J. Proakis. Digital Communications, 3rd edition, McGraw-Hill, 1995.

[8] J. Holtzman. "A simple, accurate method to calculate spreadspectrum multiple-access error probabilities". IEEE Transactions on Communications, vol. 40, no. 3, pp. 461-464, March 1992. 